\title{
Evaluation of Pap Smear And HPV VLP Serology in Clinically Suspected Cases Of Cervical Cancer in Perimenopausal Women in A Tertiary Care Centrein Kolkata
}

\author{
Raisa Ghosh ${ }^{1}$,Sumit Nandy $^{2}$,Sukla Naskar ${ }^{3}$,Baishakhi Paria ${ }^{4}$,Prof.Keya Basu ${ }^{5}$, \\ Prof. Arati Biswas ${ }^{6}$ \\ ${ }^{I}$ Department OfMedicine, GD Hospital And Diabetes Institute, India \\ ${ }^{2,3}$ Department OfPathology, Calcutta National Medical College, India \\ ${ }^{4}$ Department OfCommunity Medicine, Calcutta National Medical College, India \\ ${ }^{5}$ Department OfPathology, Calcutta National Medical College, India \\ ${ }^{6}$ Department OfGynaecologyAnd Obstetrics, Calcutta National Medical College, India
}

\begin{abstract}
Context: Carcinoma cervix is the most common genital tract carcinoma encountered among females in clinical practice in India. Pap smear is the most commonly used screening tool for early detection of cervical carcinoma. Also HPV (Human papilloma virus)VLP (Virus like particles) serology may act as an adjunctive screening procedure.

Aims and Objectives:1)To evaluate the cytological findings in clinically suspected cases of cervical cancer in perimenopausal women by conventional pap smear and correlate with biopsy findings; 2)To determine the HPV serological status and its role as a screening procedure.

Materials and Methods:Ninetyperimenopausal women, clinically suspected of having cervical carcinoma, were chosen over a one year period. They were clinically examined, relevant history taken,cytologically evaluated. All such cases were corroborated with their respective histological interpretation and were serologically evaluated for HPV VLP antibodies by ELISA (Enzyme linked immunosorbent assay).Statistical analysis:Sensitivity, specificity, accuracy, positive predictive value and negative predictive value of Pap smear were calculated, taking histopathology as the gold standard. Kappa value was determined using SPSS 18 version.
\end{abstract}

Results:Overall sensitivity, specificity, positive predictive value, negative predictive value and accuracy of conventional cytology with respect to histopathology were $78.13 \%, 96.3 \%, 92.6 \%, 88.14 \%, 89.5 \%$ respectively ( $k$ $=0.456)$. Only one case, with cervical intraepithelial neoplasia 2 (CIN2), was serologically positive for antiHPV antibodies.

Conclusion:Pap smear had moderate agreement with cervical histology in this study. However HPV VLP serology could not be established as a screening test for cervical carcinoma in perimenopausal women.

Keywords: Cervical cancer, HPV VLP Serology,PAP smear,Perimenopausal women.

Keymessages:

- Pap smear is a cost-effective screening tool with high accuracy for premalignant and malignant lesions of cervix.

- HPV VLP serology could not be established as a screening tool in perimenopausal women in cervical cancer.

\section{Introduction}

With 528,000 new cases every year, cervical cancer is the fourth most common cancer affecting women worldwide, after breast, colorectal and lung cancers; ${ }^{[1]}$ it is also the fourth most common cause of cancer death worldwide $\left(266,000\right.$ deaths in 2012). ${ }^{[1]}$ Almost $70 \%$ of the global burden falls in underdeveloped areas and more than one-fifth of all new cases are diagnosed in India. ${ }^{[1,2]}$

The Pap test (cervical cytology) is considered the most cost effective cervical cancer screening programme ever devised. ${ }^{[3]}$ Credit for its conception and development goes to George N. Papanicolaou. ${ }^{[4]}$ According to American Cancer Society, ideal time to take pap smear is 5 days after menstruation. Studies also showed that cervical carcinoma is preceded by a spectrum of intraepithelial neoplastic changes which are precancerous lesions and has been termed as Cervical intraepithelial neoplasia or CIN ${ }^{[5]} \mathrm{CIN}$ is defined as a spectrum of cellular atypia of mild degree at the base of the cervical squamous epithelium to a severe degree involving full thickness of the epithelium. ${ }^{[5]}$ The original terminology of dysplasia and carcinoma in situ was replaced by CIN, proposed by Richart, in order to emphasise the continuum of the disease ${ }^{[5]}$ Now , the Bethesda system of terminologies have been introduced to subclassify the lesions into 
grades-Low grade and High grade Squamous intraepithelial lesions(SIL). ${ }^{[5]}$ Treatment of these precancerous lesions are simple ,safe, sometimes non-destructive and usually curative ${ }^{[6]}$ Thus it is felt that an organized screening programme aimed at the high risk female population should significantly reduce the morbidity and mortality.

Further many epidemiological studies have established the role of HPV in the development of cervical cancer and its precursor lesions. ${ }^{[5]} \mathrm{HPV}$ detection increases the sensitivity of cervical cytology. ${ }^{[7]}$ The gene amplification assays like PCR is the most powerful tool for the epidemiological investigation of HPV infection or cervical cancer. ${ }^{[7]}$ It is now the gold standard for research ${ }^{[7]}$ On the other hand ,serological tests for HPV are not standardized or commercialized. ${ }^{[7]}$ However, in most studies the concordance of serological results and HPV DNA detection was reasonably high. ${ }^{[8]} \mathrm{We}$ have thus evaluated role of HPV serology in cervical cancer screening in perimenopausal women.

\section{Materials And Methods}

Approval from the institutional ethics committee was taken beforehand and an informed consent from each subject included in the study was also obtained.Perimenopausal transitional years are defined as the years prior to menopause that encompass the change from normal ovulatory cycles to cessation of menses. ${ }^{[9]}$ The average age of menopause is 51 years worldwide. ${ }^{[9]}$ In this studyperimenopausal women (40-50) attending the out-patient department of gynaecology and obstetrics and satisfying the following inclusion and exclusion criteria were evaluated, over a one year period (February 2013- January 2014). The inclusion criteria were:

- Persistent vaginal discharge

- $\quad$ Post-coital bleeding

- Intermenstrual bleeding

- Post-menopausal bleeding

- Dyspareunia

- Or,if found to have an unhealthy cervix on per speculum examination

The exclusion criteria proposed were:

- Women with previous history of vaccination against HPV

- Women with active vaginal bleeding

- Gross tumor in cervix

- Patients whose biopsy samples were not obtained

Initially 135 perimenopausal women were screened by Pap smear. However those who did not consented to the procedure of cervical biopsy were excluded from the study. Ninety perimenopausal women were, thus, selected in the study by Simple Random Sampling and a cross-sectional observational study was done. An informed consent and a detailed history regarding their socio-demographic profile was taken. Materials for Pap smear was obtained with an AYRE'S SPATULA and endocervical brush from both ectocervix and endocervix and were immediately fixed in $95 \%$ ethyl alcohol fixative for 45 minutes. Four milliliters of blood was collected in a clot vial for HPV serology. Four quadrant biopsies were taken from the cervix and collected in $10 \%$ formal saline in the same sitting.

Following material collection, staining of cervical smear by Papanicolaou method was done. Detailed microscopic examination of cervical smears and evaluation using The Bethesda System,2001 was done. Any inadequate smears were repeated in the next visit.Tissue obtained from cervical punch biopsy was processed next and stained with Haematoxylin and Eosin for Histopathological interpretation.The frequency of various cytological and histological abnormalities were tabulated.An inter-rater reliability between cytology and histology, using kappa statistic, was performed through SPSS18 version.

Results for HPV serology was obtained next, with the help of DRGInternational,Inc.HPVIgG ELISA kit (DRG International Inc.,USA;EIA-4907). HPV VLP Serology was performed among all patients satisfying inclusion criteria irrespective of cytological status. The sera were stored at $-20^{\circ} \mathrm{C}$ for 6 months and the ELISA was performed in two batches 6 months apart.Any haemolysed or visibly hyperlipemic samples were discarded.

\section{Results}

Ninety cases, in the age range of 40-50 years were sampled from among all the perimenopausal women attending the out-patient department of gynaecology and obstetrics over a period of one year.The women were of the age range of 40-50 years. The mean age group was found to be $42.32 \pm 2.96$ years. Majority of the women were of the age group of 40-42 years.Cytological evaluation of cervical smears showed that most of the cases, that is $61.1 \%$ (55 out of 90 cases) were inflammatory. This is followed by LSIL, that is, $16.7 \%$ (15 out of 90 cases). HSIL comprised of $8.9 \%$ (8 out of 90 cases) [Figure1]. ASCUS/ASC-H and SCC were $4.44 \%$ each (each consisting of 4 cases out of 90). There were three cases of ASCUS and one case of ASC-H. All the four cases of 
squamous cell carcinoma were categorised as keratinizing type [Figure 2]. Histopathologicalinterpretation of cervical biopsies showed that $61.1 \%$ (55 out of 90 cases) had chronic cervicitis. CIN1 followed with $18.9 \%$ (17 out of 90 cases) of the cases. CIN2 comprised of $11.1 \%$ (10 out of 90 cases)[Figure 3].CIN3 and SCC comprised of $4.44 \%$ each ( 4 out of 90 cases). Also $4.44 \%$ of the cases were within normal limit (4 out of 90 cases). Thus, 35 out of 90 cases had epithelial abnormality (about 38.89\%).Overall sensitivity, specificity, accuracy of conventional Pap smear were $78.13 \%, 96.3 \%$ and $89.5 \%$ respectively [Table 1]. Positive predictive value and negative predictive value were $92.6 \%$ and $88.14 \%$ respectively [Table 1].

All the above calculations were done excluding ASCUS/ASC-H cases and patients with 'inflammatory' smears and 'within normal limit' were categorized as non-diseased population.There was moderate or intermediate degree of agreement between cytological and histological interpretation. The interrater variability for the raters(cytology vs histology) or kappa value was found to be 0.456 [Table 2], which is interpreted as 'moderate agreement' between cytology and histology of cervical lesions. Only one sample was reactive for HPV IgG ELISA (89 samples were non-reactive). The reactive sample was that of a 50 year old woman with a CIN2 histological diagnosis (inflammatory smear on cytology).

\section{Discussion}

Cervical cancer is preceded by a distinct latent period of more than a decade. ${ }^{[11]}$ This interval between preinvasive disease (CIN) and cervical cancer provides us with the opportunity to prevent cervical cancer through screening. Most of the cases with preinvasive disease predominate in the fourth and fifth decade of life. ${ }^{[12]}$ So in this present study we have chosen the fifth decade of life, that is, the perimenopausal age group, where both the rate of abnormal smears and invasive cancers are higher than in any age group. ${ }^{12,13]}$

Tamboli et al recorded the prevalence of inflammatory smears as $50.91 \%$, LSIL as $14.55 \%$, HSIL as $10.9 \%$ and ASCUS as $8.64 \% .{ }^{[14]}$ However they reported a higher prevalence of squamous cell carcinoma $(15 \%)$ than the present study $(4.44 \%){ }^{[14]}$

Saha et alalso reported the prevalence of ASCUS, LSIL, HSIL,SCC and Benign lesions as $2.32 \%, 18.6 \%, 20.9 \%, 6.9 \%$ and $51.16 \%$ respectively. ${ }^{[15]}$ When compared to the present study, the prevalence of HSIL is found to be markedly higher $(8.9 \%$ in the present study). Like the present study, inflammatory smears constituted the majority of the smears.

Bal et al recorded the prevalence of ASCUS,LSIL, HSIL,SCC as $0.3 \%, 2.7 \%, 0.7 \%, 1.3 \%$ respectively. ${ }^{[16]}$ Majority of the smears were inflammatory $(74.3 \%)$ which is similar to that of our study. ${ }^{[16]}$ However, the epithelial cell abnormalities constituted only about 5\% of the smears unlike $38.89 \%$ derived from our study. ${ }^{[16]}$ A possible explanation may be due to screening of a high risk group like perimenopausal women in the present study as compared to the aforesaid study.

Abaliet al recorded the prevalences of CIN1, CIN2, CIN3, and SCC as 22.7, 3.7, 9 and 7.4\% respectively. ${ }^{[17]}$ The prevalence of the non-neoplastic group histologically was $56.9 \% .{ }^{[17]}$ The prevalence of CIN2 is lower and CIN3 is higher than the present study. However the net prevalence of CIN2 and CIN3 taken together is similar to the present study. The prevalence of CIN1 and the non-neoplastic group corroborated with the present study.

Katz et al recorded the prevalence of CIN 1,CIN2, CIN3, and SCC as $21 \%, 21.3 \%, 18 \%$ and $2.4 \%$ (including both invasive and microinvasive squamous cell carcinoma) respectively. ${ }^{[18]}$ The prevalence of CIN1 in the present study is the same as above. However, the prevalence of CIN2 and CIN3 were much higher than the present study. This may be due to the larger sample size of 334 women ${ }^{[18]}$ as compared to 90 women in our study. Secondly, the women in the above study underwent colposcopic directed biopsy which may also increase the sensitivity of histopathology. Also the proportion of women with squamous cell carcinoma is lower than in the present study.

The diagnostic accuracy of cytology or Pap smear in the present study $(89.5 \%)$ was higher than the other studies. Nawaz FH et al in 2005 reported an accuracy of $74 \% .{ }^{[19]}$ Anschau et alreported as $69.8 \%$ in $2011 .{ }^{[20]}$ Tamboli et al in 2014 reported accuracy of cervical cytology as $74.09 \%$. ${ }^{[14]}$

Anschauet al in 2011 showed kappa value of 0.498 when cytology and histology results were matched according to The Bethesda 2010, showing moderate degree of agreement between histology and cytology, which corroborated with the present study. ${ }^{[0]}$

However, Katz et al in 2012 found the kappa value for conventional cervical cytology screening as 0.261 , representing fair agreement. ${ }^{[18]}$ This may be due to interpretation error or sampling error.

Further there was only one reactive sample, so no association between HPV serology and cervical dysplasia/cancer could be obtained. Thus, the aim of this study to evaluate HPV serology as a screening tool was not fulfilled.

Rama et al found that the prevalence of positive serology for anti HPV antibodies in healthy young women with an abnormal cytology was only 1.75 times that of abnormal cytology and negative 
serology ${ }^{[21]}$ Thus, they concluded that serological results showed no association with abnormal cytological results. ${ }^{[21]}$ This observation is consistent with the present study.

Achour et al detected HPV-specific antibodies in sera of 60 women, with inflammatory Pap smears, of reproductive, perimenopausal and post-menopausal age group. ${ }^{[2]}$ Results showed that six patients $(10 \%)$ (showed a positive reactivity to at least one of the HPV-16 or HPV-18 antigens and sera showed different reactivity to the different antigens with the following percentages: 5\%, 3\%, 2\%, 3\% and 3\% for L1 HPV-16, E6 HPV-16, E7 HPV-16, E6 HPV-18 and E7 HPV-18 respectively. ${ }^{[22]}$ They had used GST capture ELISA for measuring anti-HPV antibodies, ${ }^{[22]}$ unlike VLP L1 ELISA used in the present study. They concluded that Serology is not in itself a diagnostic method but must be kept in consideration for monitoring patients with an inflammatory cervical smear. Similarly, the determination of these antibodies by GST-capture ELISA and Luminex can help monitor vaccination programs on a large scale. ${ }^{[22]}$

Yepinget al in 1999 evaluated the serological assays for HPV as screening tests for invasive cervical cancer . Serum samples were collected from 194 patients of cervical cancer. ${ }^{[23]}$ HPV-16 VLP serology had a sensitivity of $47.4 \%$ and a specificity of $75.6 \%$, whereas HPV-16 E6 and E7 serology had a sensitivity of $63.4 \%$ and a specificity of $89.9 \% .{ }^{23]}$ Thus VLP ELISA detected only half of all the cases (Total $n=194$ ) of invasive cervical cancer. ${ }^{[23]}$ The present study, however, had only 4 cases of invasive cervical cancer which did not show any detectable titre of antibodies.

Wideroff et al, however showed in their nested case control study of cervical intraepithelial neoplasia that overall, $30 \%$ of the patients with cervical intraepithelial neoplasia and $6.6 \%$ of the controls (without Cervical intraepithelial neoplasia) were positive for multiple types of HPV. ${ }^{[24]}$ The discrepancy with our study can be explained by the following reasons:

- Number of cases with CIN were 80 in the study done by Wideroff et al unlike 31 in the present study.

- The above cited study included women of reproductive, perimenopausal and post-menopausal age group, unlike only perimenopausal women in the present study. And it is recorded that seroprevalence is more in women of reproductive age group. ${ }^{[7]}$

This study was undertaken to determine the diagnostic accuracy of cervical cytology taking histopathology as the gold standard in perimenopausal women and to evaluate HPV VLP serology as a screening tool for cervical cancer, in addition to cervical cytology.Pap smear corroborated well with cervical histopathology. However, role of HPV VLP serology as a screening tool for cervical cancer could not be justified.

\section{There can be two possible explanations for the above observation regarding HPV serology:}

First, the sample size was much smaller than in similar studies.Secondly, it is well documented that serum anti-HPV antibody development requires a prolonged median lag period of $\sim 12$ months from the first detection of HPV DNA, and the titer of detectable serum antibodies has been low (maximal, 1/200). ${ }^{[25]}$ However, another fact of prime importance is that anti-VLP antibodies do not persist for years after infection. ${ }^{[7]}$ In fact, sero-epidemiological data suggest that they disappear with age ${ }^{[7]}$ The age group included in the present study is that of women in the fifth decade, when any seroconversion which may have taken place several years back, wanes off. This may be a reason for obtaining no detectable titre of anti-HPV antibodies in majority of the cases.

\section{References}

[1]. Ferlay J, Soerjomataram I, Ervik M, Dikshit R, Eser S, Mathers C et al. GLOBOCAN 2012, Cancer Incidence and Mortality Worldwide. International Agency for Research on Cancer; Lyon,France:2013.

[2]. Bray F, Ren JS, Masuyer E, FerlayJ.Global estimates of cancer prevalence for 27 sites in the adult population in 2008. Int J Cancer 2013; 132:1133-45.

[3]. Janicek MF, Averette HE: Cervical cancer: Prevention, diagnosis, and therapeutics. CA Cancer J Clin 2001;51:92-114.

[4]. Papanicolaou GN, TrautAF.The diagnostic value of vaginal smears in carcinoma of the cervix. Am J ObstetGynecol 1941;42:192206.

[5]. Koss LG, Melamed MR, editors. Koss' Diagnostic cytology and its histologic bases.5th ed. New York: Lippincott Williams and Wilkins; 2006. 306-08p.

[6]. Goodman A. Role of routine human papillomavirus subtyping in cervical screening. GynecolOncol1961;34:221-29.

[7]. Coutlee F, Rouleau D, Ferenczv A, Franco E. The Laboratory Diagnosis of Genital Human Papillomavirus infections. Can J Infect Dis Med Microbiol 2005; 16:83-91.

[8]. Dillner J. The serological response to papillomaviruses. Semin Cancer Biol 1999;9:423-30.

[9]. Fritz MA, Speroff L. Clinical Gyneacologic Endocrinology and Infertility. $8^{\text {th }}$ ed. Wolters Kluwer, Lippincott Williams and Wilkins ; 2011.673-748 p.

[10]. McHugh ML. Interrater reliability: the kappa statistic. Biochem Med (Zagreb) 2012; 22:276-282

[11]. Forsmo S, Jacobsen BK, Stalsberg H. Cervical neoplasia in PAP smears: Risk of cervical intraepithelial neoplasia (CIN) after negative or no prior Smears in a Population without a Mass Screening Programme. Int. J. Epidemiol 1996; 25. 
[12]. Gupta S, Sodhani P, Halder K, Chachra KL, Singh V, Sehgal A. Age trends in pre-cancerous and cancerous lesions of the uterine cervix in a cytology screening programme: what should be the target age group for a major thrust of screening in resource-limited settings? Cytopathology 2008; 19:106-10.

[13]. Neighbor RM, Newman RL. Incidence of cervical cancer in perimenopausal and postmenopausal women detected by Papanicolaou smears. Am J ObstetGynecol 1976; 124:348-51.

[14]. Tamboli GD, Khatod LV. Accuracy of cytological findings in abnormal cervical smear by cyto-histological comparison. Journal of Medical Education and Research 2013; 3.

[15]. Saha R, Thapa M. Correlation of cervical cytology and cervical histology. Kathmandu Univ Med J(KUMJ) 2005;3:222-4.

[16]. Bal MS, Goyal R, Suri AK, Mohi MK. Detection of abnormal cervical cytology in Papanicolaou smears. J Cytol 2012;29:45-7.

[17]. Abali R et al. Histopathological correlation of squamous cell abnormalities detected on Cervical cytology. Turk PatolojiDerg2011;27:144-8.

[18]. Katz LMC, Souza ASR, GuerraJMDM,Amorim MMR. Is it necessary to repeat cervical cytology at the tie of a colposcopy? Brazilian Journal of Mother and Child Health 2012; 12.

[19]. Nawaz FH, Aziz AB, Perwez S, Rizvi JH .Prevalence of abnormal papanicolaou smears and cytohistological correlation. A study from Aga Khan University hospital, Pakistan. Asia Pac J ClinOncol 2005;1:128-32.

[20]. Anschau F, Goncalves MAG. Discordance between cytology and biopsy histology of cervix: what to consider and what to do. ActaCytol2011;55:158-62.

[21]. Rama CH, Roteli-Martins CM, Derchain SF, Oliveira EZ, Aldrighi JM, MarianiNeto C. Serological detection of anti-HPV 16/18 and its association with Pap smear in adolescents and young women. Rev Assoc Med Bras 2006; 52:43-7.

[22]. Achour M, Zeghal D. Cervical Cancer in Women with Inflammatory Pap Smears. Journal of Cancer Therapy 2014; 5:82-90.

[23]. Sun Y,Eluf-Neto J, Bosch FX, Muñoz N, Walboomers JM, Meijer CJ et al.Serum Antibodies to Human Papillomavirus 16 Proteins in Women from Brazil with Invasive Cervical Carcinoma.CancerEpidemiol Biomarkers Prev 1999; 8:935.

[24]. WideroffL, Schifmann M, Haderer P, Armstrong A, Greer CE, Manos MM et al. Seroreactivity to Human Papillomavirus Types 16, 18, 31, and 45 Virus Like Particles in a Case-Control Study of Cervical Squamous Intraepithelial Lesions. J Infect Dis 1999;180:1424-8.

[25]. Hagensee ME, Koutsky LA, Lee SK, Grubert T, Kuypers J, Kiviat NB et al. Detection of Cervical Antibodies to Human Papillomavirus Type 16 (HPV-16) Capsid Antigens in Relation to Detection of HPV-16 DNA and Cervical Lesions. J Infect Dis 2000; 181:1234-9.

Tables:

Table 1: Correlation Between Cytological And Histological Interpretation ( $\mathrm{N}=90)$

\begin{tabular}{|c|c|c|c|c|c|c|c|}
\hline & & \multicolumn{5}{|c|}{ Histological Diagnosis } & \multirow[b]{2}{*}{ Total } \\
\hline & & $\mathrm{CC}+\dagger$ & CIN1t+ & CIN2 & CIN3 & $\mathrm{SCC}$ & \\
\hline \multirow{7}{*}{$\begin{array}{l}\text { Cytological } \\
\text { Diagnosis }\end{array}$} & $\mathrm{Wnl} *$ & 3 & 1 & _ & $\ldots$ & _ & 4 \\
\hline & $\operatorname{Inflm} \uparrow$ & 49 & 4 & 2 & 0 & - & 55 \\
\hline & $\begin{array}{l}\text { Ascus } \$ / \\
\text { Asc-H§ }\end{array}$ & 1 & 1 & 2 & - & - & 4 \\
\hline & Lsil\| & 2 & 9 & 3 & 1 & $=$ & 15 \\
\hline & Hsil| & - & 2 & 3 & 3 & - & 8 \\
\hline & $\mathrm{Scc}^{* *}$ & $\ldots$ & $\ldots$ & $\ldots$ & $\ldots$ & 4 & 4 \\
\hline & Total & 55 & 17 & 10 & 4 & 4 & 90 \\
\hline
\end{tabular}

Note: *WNL, Within normal limit; †INFLM, Inflammatory; $\neq$ ASCUS, Atypical squamous cells of undetermined significance; \$ASC-H, Atypical squamous cells-cannot exclude HSIL ; \|LSIL, Low grade squamous intraepithelial lesion; IHSIL, High grade squamous intraepithelial lesion; **SCC, Squamous cell carcinoma, $\dagger$ †C, Chronic cervicitis; $\nrightarrow$ CIN, Cervical intraepithelial neoplasia.

Table 2: Measurement Of Kappa Value

\begin{tabular}{|c|c|c|c|c|c|c|}
\hline \multicolumn{7}{|c|}{ Cyto - HistoCrosstabulation } \\
\hline \multicolumn{7}{|c|}{ Count } \\
\hline & & \multicolumn{4}{|c|}{ Histo } & \multirow{2}{*}{ Total } \\
\hline & & 1 & 2 & 3 & 4 & \\
\hline \multirow{5}{*}{ Cyt } & & & & & & \\
\hline & 1 & 9 & 4 & 2 & 0 & 15 \\
\hline & 2 & 2 & 6 & 0 & 0 & 8 \\
\hline & 3 & 5 & 2 & 52 & 0 & 59 \\
\hline & 4 & 0 & 0 & 0 & 4 & 4 \\
\hline \multicolumn{2}{|c|}{ Total } & 16 & 12 & 54 & 4 & 86 \\
\hline
\end{tabular}

Note: Cyto: 1-LSIL, 2-HSIL, 3-Inflammatory+Within Normal Limit, 4-Squamous Cell Carcinoma; Histo: 1CIN1, 2-CIN2+CIN3, 3-Chronic Cervicitis, 4- Squamous Cell Carcinoma 


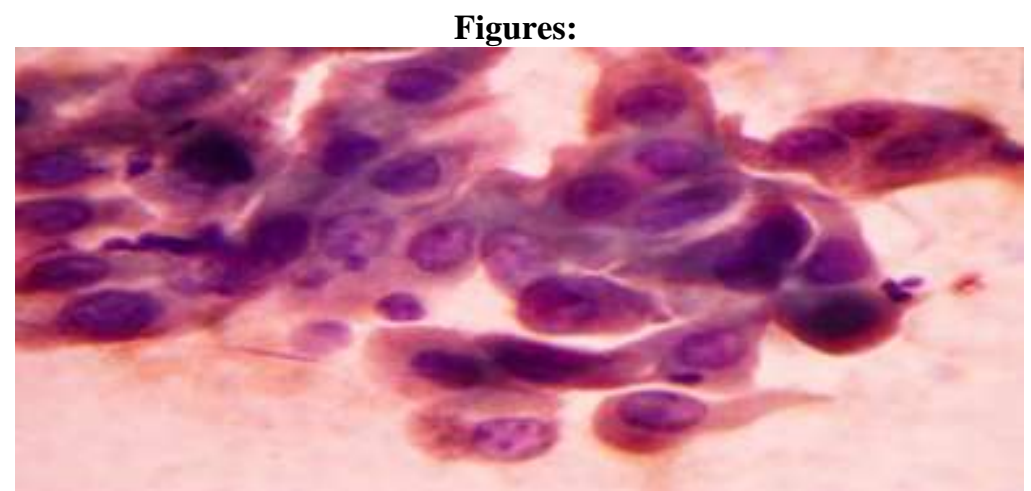

No.1. HSIL/High grade squamous intraepithelial lesion; Pap stain (100x)

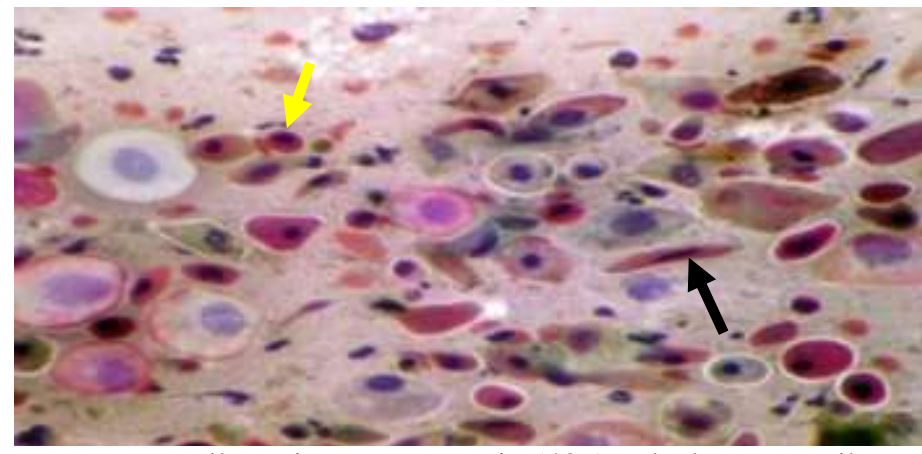

No.2. Keratinising squamous cell carcinoma; Pap stain (40x); Black arrow- Fibre cell, Yellow arrowdyskeratotic cell

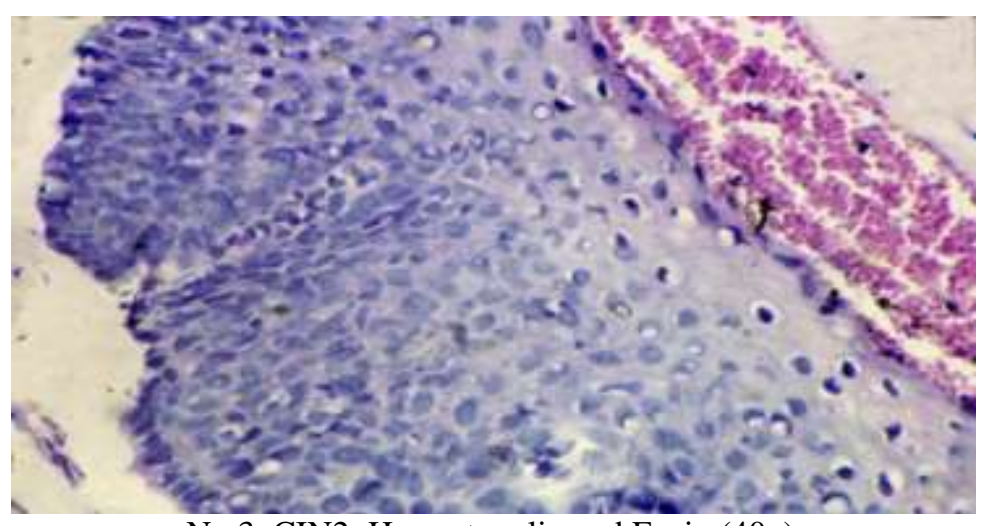

No.3. CIN2; Haematoxylin and Eosin (40x)

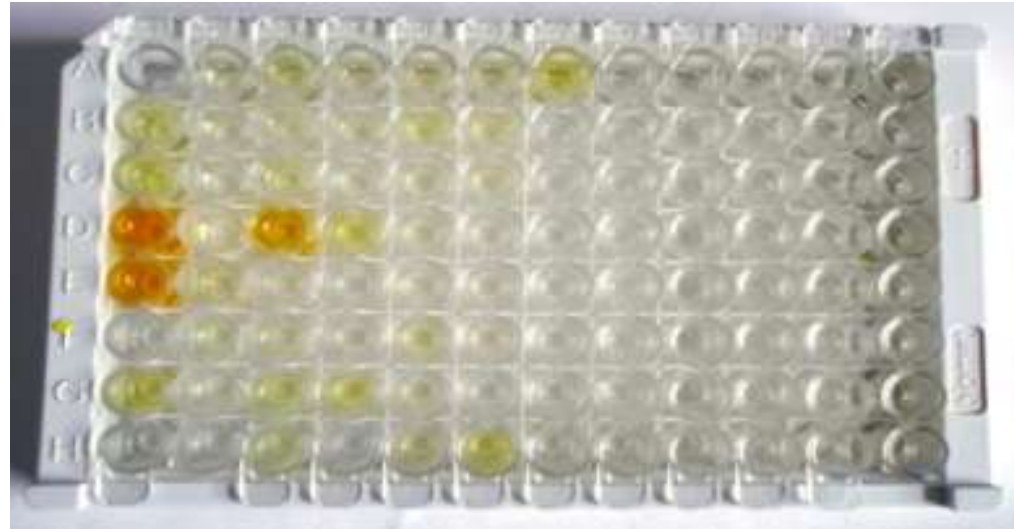

No.4. IgG HPV ELISA Microplate showing two positive controls and one reactive sample (dark yellow colour in third vertical row) 